\title{
Optical study of swirl during combustion in a CI engine with different injection pressures and swirl ratios compared with calculations.
}

\author{
Henrik W. R. Dembinski, Hans-Erik Ångström \\ Scania CV AB, Royal Institute of Technology Stockholm
}

Copyright $@ 2011$ SAE International

\begin{abstract}
Spray and mixture formation in a compression-ignition engine is of paramount importance in the diesel combustion process. In an engine transient, when the load increases rapidly, the combustion system needs to handle low $\lambda$ operation without producing high $\mathrm{NO}_{\mathrm{x}}$ emissions and large amounts of particulate matter. By changing the in-cylinder flow, the emissions and engine efficiency are affected. Optical engine studies were therefore performed on a heavy-duty engine geometry at different fuel injection pressures and inlet airflow characteristics. By applying different inlet port designs and valve seat masking, swirl and tumble were varied. In the engine tests, swirl number was varied from 2.3 to 6.3 and the injection pressure from 500 to 2500 bar. To measure the in-cylinder flow around TDC, particle image velocimetry software was used to evaluate combustion pictures. The pictures were taken in an optical engine using a digital high-speed camera. Clouds of glowing soot particles were captured by the camera and traced with particle image velocimetry software. The velocity-vector field from the pictures was thereby extracted and a mean swirl number was calculated. The swirl number was then compared with 1D simulation program GT-POWER and CFD based correlations. The GT-POWER simulations and CFD based correlation calculations were initiated from steady-state flow bench data on tested cylinder heads.

The main conclusions from this study were that the mean swirl numbers, evaluated with the PIV software from combustion pictures around TDC, agreed with CFD based correlations and the low swirl numbers also correlated with the 1D-simulation program. Most of the induced swirl motion survives the compression and combustion, while the induced tumble does not survive to the late combustion phase. The tumble however, disturbs the swirl motion and offsets the swirl centre. This offset survives the compression and combustion. The diesel sprays that are injected symmetrically in the combustion chamber are thereby exposed to the swirl asymmetrically. This study also shows that the angular velocity at different piston bowl radii deviates from solid body rotation. The angular velocity is higher closer to the centre and decreases to be at the lowest value at the outer piston bowl edge. When the injection pressure is increased, the deviation from solid body rotation increases due to spray effects.
\end{abstract}

\section{INTRODUCTION}

The importance of intake airflow in diesel engines is well established, and many heavy-duty (HD) diesel engines have a tangential swirl motion to improve combustion. The swirl flow has been proven to reduce particulate matter (PM) emissions from the engine and much research on swirl flow has been carried-out for a long period of time. Lately, the main focus of the research has been on injection systems with extremely high injection pressures and, in many cases, with a quiescent combustion chamber (with no, or nearly no, swirl in the cylinder). The higher injection pressure improves droplet break-up, air/fuel mixing in the spray [1] and increases turbulent intensity in the combustion chamber. This is of paramount importance, especially during an engine transient, when the combustion system needs to handle low air/fuel ratio $(\lambda)$ conditions without producing extremely high PM, especially for engines without diesel particulate filters (or other PM-reducing after-treatment). With swirl, the PM can be greatly reduced [2] under a transient, even when high injection pressures are used. 
The two main flow structures in the cylinder, swirl number (SN) and tumble, are defined as:

$$
\begin{aligned}
& S N=\frac{\omega_{\text {Swirl }}}{\omega_{\text {Engine }}} \\
& \text { Tumble }=\frac{\omega_{\text {Tumble }}}{\omega_{\text {Engine }}}
\end{aligned}
$$

where

$\omega_{\text {Swirl }}=$ air rotational velocity around cylinder centre axis

$\omega_{\text {Tumble }}=$ air rotational velocity perpendicular to the cylinder axis

$\omega_{\text {Engine }}=$ engine rotational crankshaft velocity

Port designs on diesel engines have historically been very important [3], [4]. SN has been an important factor for a good combustion and low PM. To create an understanding of how the in-cylinder flow behaves, particle image velocitmetry (PIV) measurements are commonly used in both constant flow rigs and motored engines. In [5], PIV measurements were done from inlet stroke until close to firing TDC and compared with CFD calculations on the same engine geometry as used in this paper. The main conclusion was that, before TDC, the swirl centre moves around and does not coincide with the geometrical cylinder centre. The swirl centre axis was also observed to be tilted since it did not have the same position at two different distances from the cylinder head at a certain crank angle degree (CAD). The main trend with measured and CFD calculated SN is the same, even if there is a deviation between the two methods. Other papers also show the swirl centre offset before TDC and the movement of the swirl centre at a later CAD [6]. Incylinder flow is complex and cycle-to-cycle variations are inevitable, due to its turbulent nature. In [7], it was observed that the SN was fluctuating at different cycles which were repeated after each other. The SN also differs depending on how close to the fire deck the measurement is done [8]. In [9], PIV measurements were combined with CFD simulations and optical spray images. PIV measurements were done in the early injection phase (during the ignition delay) at different SN. With a higher SN more fuel droplets were concentrated in the centre region of the combustion chamber [9] due to shorter penetration. In [2] tests with up to 2000 bar injection pressure at $\lambda 1.2$ and load of 10 bar indicated mean effective pressure (IMEP) were performed. The emissions were greatly affected when SN and tumble were changed. To examine why the emissions were so greatly affected at different flow structures, optical engine tests were performed in this work. PIV software was used to calculate the flow characteristics from the pictures taken in the optical engine after the injection had ended. The $\mathrm{SN}$ was varied from 2.3 to 6.3 and tumble from 1 to 2.2 , as defined in equations (1) and (2).

\section{TEST SETUP}

The test points of interest were chosen from a measured engine transient at $1000 \mathrm{rpm}$, which can be seen in Figure 1 where "Req. Torque" is the requested engine load from the operator. The transient, from 3 to 23 bar IMEP, was performed on a six-cylinder engine with a similar combustion system as in the optical engine. The three points of interest, seen in Table 1 , were investigated further in the optical engine, with the same boundary conditions as in the six-cylinder engine, they were tested in steady-state conditions.

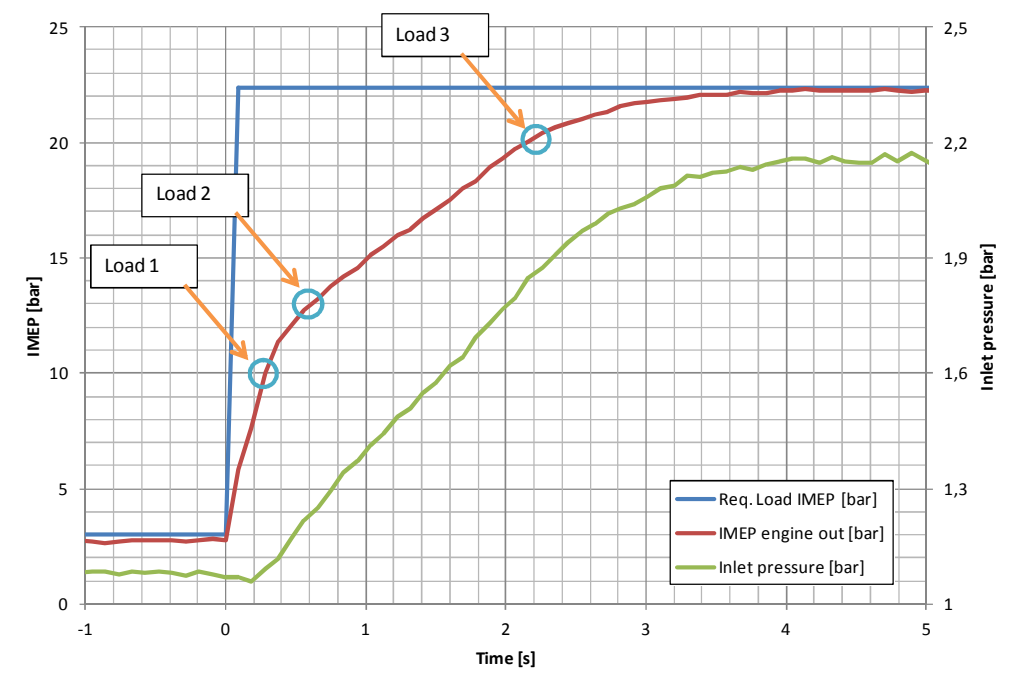

Figure 1 Engine load build up during a transient, from low (3 bar IMEP) to full load (23 bar IMEP). 
Load point 1 is the same as tested in [2] and corresponds to a maximum natural aspirated engine load, 10 bar IMEP, with injection pressures from 500 to 1500 bar in 500 bar increments, and $\lambda=1.25$. The fuel mass was kept constant by changing the injection duration for the different injection pressures and crank angle for maximum cylinder pressure (APmax) was kept constant at $11^{\circ}$ ATDC by adjusting the start of injection (SOI). The SN was varied from 2.3 to 6.3 by using two different cylinder heads and by blocking (or not) an inlet port. To maintain a constant $\lambda$, the boost pressure was adjusted slightly to maintain the same air mass flow into the engine when the inlet geometry was varied. Load point 2 was performed at 2000 bar injection pressure and a slightly higher load, 13 bar IMEP, when the boost pressure starts to increase on a normal turbo-charged DI engine. Only the cylinder head with high SN was used in load points 2 and 3. Load point 3 was performed at 20 bar IMEP with $\lambda=1.1$ and is the point before the boost pressure reaches steady-state conditions.

\begin{tabular}{|c|c|c|c|}
\hline & Load $1 \mathrm{a}, \mathrm{b}, \mathrm{c}$ & Load 2 & Load 3 \\
\hline Rail press. [bar] & $500,1000,1500$ & 2000 & 2500 \\
\hline $\mathrm{SN}$ (calc) at BDC & $2.3-6.3$ & $3.4 \& 6.3$ & $3.4 \& 6.3$ \\
\hline Tumble(calc) at BDC & $1-2.2$ & $1.1 \& 2.2$ & $1.1 \& 2.2$ \\
\hline SOI ATDC & $-11^{\circ},-6^{\circ},-4^{\circ}$ & $-3^{\circ}$ & $-2^{\circ}$ \\
\hline IMEP & 10 bar & 13 bar & 20 bar \\
\hline
\end{tabular}

The optical test engine, used in this study, is based on a Scania engine geometry and injection system capable of 2500 bar injection pressure, see Figure 2 and Table 2. On the original piston, a piston extension is mounted that leads to the optical piston and the liner that it is fitted into. A high-speed colour camera, Phantom v7.3, is installed next to the engine and the combustion light is transferred to the camera through a mirror mounted in the piston extension. For more information on the optical engine, see [10].

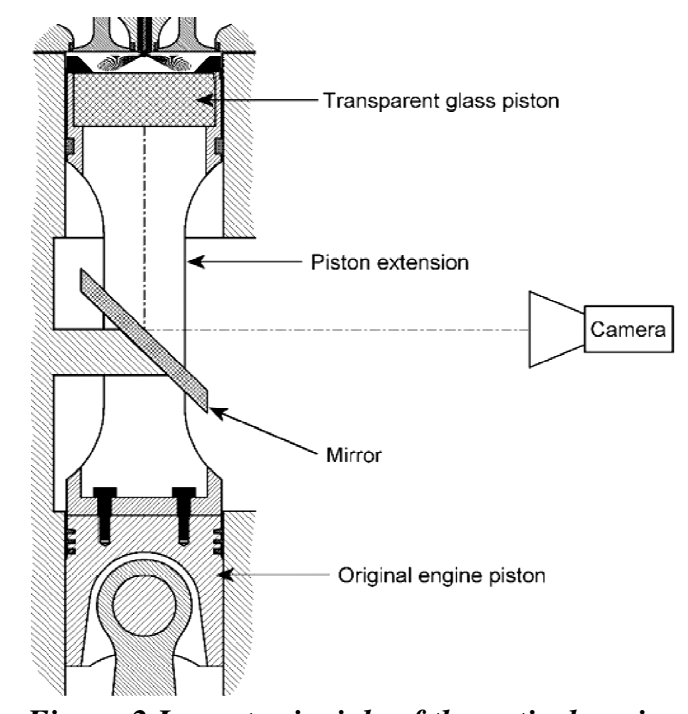

Figure 2 Layout principle of the optical engine.

Table 2 Optical engine specifications.

\begin{tabular}{|c|c|}
\hline Test engine & 4-stroke Scania D12 \\
\hline Bore/stroke [mm] & $130 / 154$ \\
\hline Connecting rod [mm] & 255 \\
\hline Compression ratio & $17.3: 1$ \\
\hline No. of valves & 4 \\
\hline Injection system & Scania common rail XPI \\
\hline Injector holes & 8 \\
\hline $\begin{array}{c}\text { Spray angle [deg] } \\
\text { (obetween cyl.head and spray) }\end{array}$ & 16 \\
\hline $\begin{array}{c}\text { Injector hole diameter } \\
\text { (inner/outer) [mm] }\end{array}$ & $0.187 / 0.163$ \\
\hline Max Injection pressure [bar] & 2500 \\
\hline
\end{tabular}

The two different piston bowls tested in this study are seen in Figure 3, one with flat glass and one with bowl-shaped glass. The flatglass piston bowl is used as no compensation needs to be done for the refractive index and it's the most common geometry in optical

Page 3 of 17 
engines. The bowl-shaped glass, on the other hand, is more like the real engine configuration, and comparison with the flat piston bowl is thereby possible if both geometries are used. The glass is mounted in a titanium piston that transfers the combustion pressure on the glass to the piston extension. The piston extension is compressed by $1.5 \mathrm{~mm}$ at 160 bar cylinder pressure and decreases the experienced compression ratio. To compensate for the lower compression ratio, the boost pressure and inlet temperature was increased so the motoring cylinder pressure in the optical engine was equal to the six-cylinder engine. The $\lambda$ was slightly higher in the optical engine compared with the six-cylinder engine. The increase of inlet temperature was also done to compensate the increased ignition delay in the optical engine, as only one combustion event was performed under the measurement. A titanium clamping ring was mounted above the piston glass to fix it. This restricted the field of vision to a diameter of $80 \mathrm{~mm}$, compared with the total cylinder bore of $130 \mathrm{~mm}$.

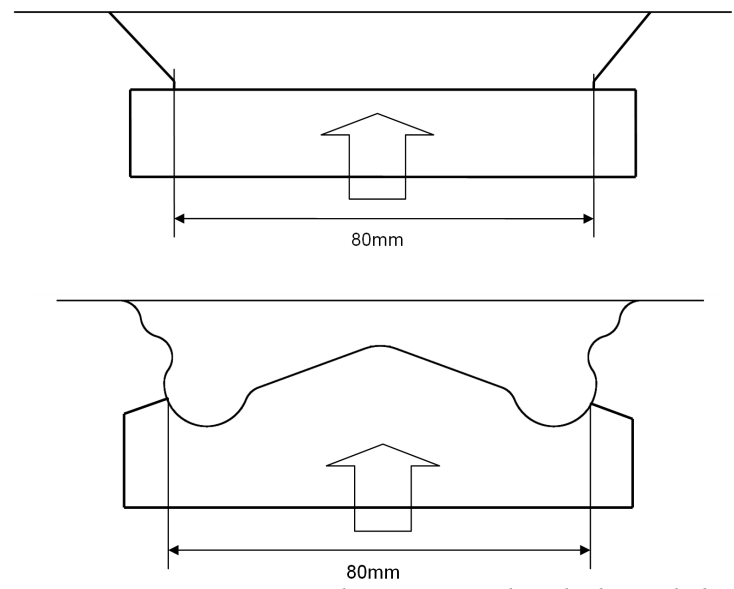

Figure 3 Tested piston bowls, flat piston, (top picture), and re-entrant bowl-shaped (bottom picture). The arrows show the observer's view into the combustion chamber.

To be able to vary the in-cylinder airflow, two different cylinder heads were used in this study (named low and high SN head). Each head was tested with one or two operating inlet ports, called v1 and v2, respectively, in this report, to further extend the variation of the in-cylinder airflow. Both inlet valves are operated in both cases and one port is blocked with a solid plate mounted between the cylinder head inlet port and inlet runner in one of the cases. When using one port, the SN and tumble number increased compared with using two ports. It has been shown, in [2], that this can affect the ignition delay. In [11], a self-ignition model was developed with turbulent mixing during the self-ignition event implemented in the model. The performed combustion bomb tests indicate the impact on the self-ignition event when the turbulent intensity is changed, in this case with different injection pressures.

One of the cylinder heads used is seen in Figure 4. It has two separate inlet ports (indicated by blue arrows) with extra masking over the valve seat (see the red circles in Figure 4b). The masking over the valve seat was used to generate a higher SN compared with the standard head that did not have the extra masking. The standard head had the same layout as the high SN-head, apart from the masking. The generated swirl flow direction is defined in Figure 4a (clockwise, seen from below) for all readings in this report.

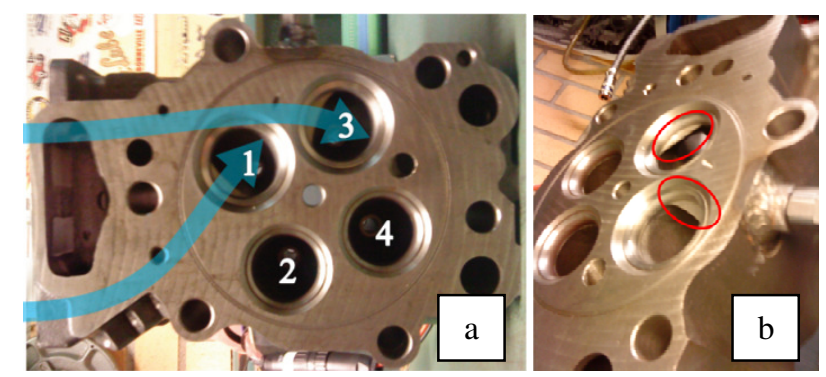

Figure 4 Orientation of the valves and the inlet ports on the tested cylinder head. The inlet valve seats have extra masking for the high SN head, marked in picture b, to increase the swirl.

The exhaust back pressure was set equal to the inlet pressure (1:1) in all the test points. The cooling water and oil temperatures were stabilized at $90^{\circ} \mathrm{C}$. SOI used in this report is the start of the electrical signal that is sent to the XPI common rail injector. The SOI delay and the end of injection, in ms, between the electrical signal and the fuel injection are different depending on injection pressure. For more details, see study [12].

Page 4 of 17 


\section{CALCULATIONS OF SWIRL AND TUMBLE}

Calculations of swirl, tumble and turbulent intensity were performed in GT-POWER. GT-POWER data for airflow calculations was measured in a constant flow-rig, where the swirl and tumble were measured as a function of valve lift. For more details, see [2]. The normalised turbulent intensity is a global mean parameter for the entire cylinder, and values shown in the tables are at cycle start 100 CAD before top dead centre ( ${ }^{\circ}$ BTDC), calculated in GT-POWER with a k- $\varepsilon$ based model. The normalised turbulent intensity (NTI) is defined as:

$$
\mathrm{NTI}=\frac{u^{\prime}}{V_{p}}
$$

$u^{\prime}=$ Turbulent intesity $[\mathrm{m} / \mathrm{s}]$

$V_{p}=$ Mean velocity of the piston $[\mathrm{m} / \mathrm{s}]$

How GT-POWER calculates the above-mentioned in-cylinder flow can be seen in [13], [14] and [15]. In GT-POWER, the swirl, tumble and NTI can be plotted as a function of CAD. During the intake stroke, the mean swirl and mean tumble build up. The inflow values are functions of valve lift, according to the rig test data. At inlet valve closure (IVC), the tumble and swirling vortex are assumed to be conserved in the cylinder. The air rotation accelerates during compression when the swirling air is forced into the piston bowl, due to the geometric change. The turbulent intensity is high during the inlet stroke when the airflows into the cylinder at a high velocity. The turbulent intensity drops during compression, caused by dissipation of the in-cylinder gas fluid, and close to TDC it starts to increase again when the tumble vortex is transferred into turbulence with a smaller length scale. This behaviour has been observed in large eddy simulations (LES) in [16]. Near TDC, the distance between piston and cylinder-head at the squish area is rapidly changing, creating a squish flow that also increases the turbulent intensity at TDC. This has been observed in GT-POWER simulations shown in [2]. Close to TDC the fuel injection creates high turbulent intensity in the cylinder. The turbulent eddies dissipate quickly to smaller length scales (and in the end to heat) after the injection has ended. In Table 3 and Table 4 the calculated SN and tumble are shown at different CAD at load point 1c for all tested hardware combinations. It is clear to see that GT-POWER estimates a higher SN at TDC compared with BDC in all of the cases. After TDC the SN decreases. This seems to be the logical behaviour, when the added fuel in the cylinder needs to be accelerated with the conserved momentum in the swirling air together with shear layer stress from the boundary layer and geometry change during the expansion.

Table 3. Calculated SN in GT-POWER at different CAD for load point 1c, 1500 bar injection pressure.

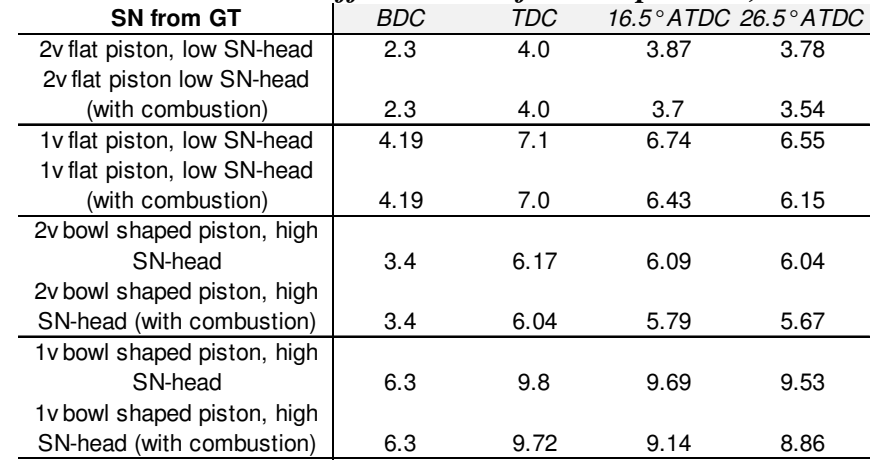

Table 4. Calculated tumble in GT-POWER at different CAD for load point 1C, 1500 bar injection pressure.

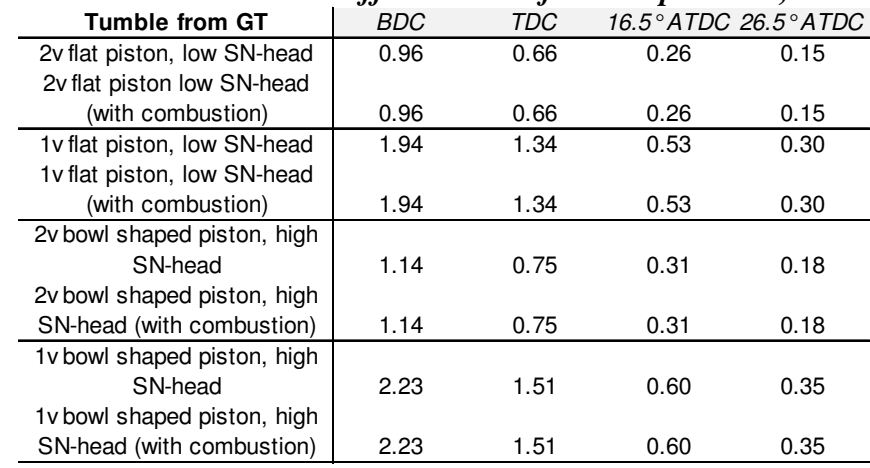


In [17], CFD calculations were used, together with constant flow-rig measurements, to calculate the SN at TDC. An empirical expression was derived, reproduced here in equation 4. To compare their results with the model in GT-POWER and later in this paper with the PIV results, their model was used for our specific case. The BDC SN was taken from GT-POWER calculations and the $S N_{T D C}$ is defined as [17]:

$$
S N_{T D C}=\frac{r_{D}^{2} \cdot M S N}{\beta_{1}+\beta_{2} \cdot r_{D}^{2} \cdot M S N}
$$

where

$M S N=$ mean swirl number $(\mathrm{BDC})$

$$
r_{D}=\frac{\text { Cylinder radius }}{\text { Piston bowl radius }}
$$

$\beta_{1}=1.1594$

$\beta_{2}=0.1209$

Coefficient $\beta_{1}$ represents the difference between steady-state SN and SN calculated by the CFD method shown in [17]. $\beta_{2}$ is a factor coupled to the friction losses. The full comparison of the different calculations and measurements can be found in Table 5, at the end of this paper.

\section{Evaluation of combustion pictures}

To compare the 1D-simulation results, combustion pictures were taken in the optical engine with a high-speed Phantom camera. The pictures were then evaluated with PIV software, DaVis 7.2, were velocity vector fields were extracted from the moving combustion cloud. Normal PIV measurements during combustion in a diesel engine are difficult. The seeding, particles that are introduced in the inlet air can be combusted or destroyed by the high-temperature combustion. The introduced laser sheet in the cylinder can easily be drowned in the bright combustion light that contains broad spectrum of wavelengths. The flow in the cylinder is three dimensional and turbulent. To catch the turbulent phenomena, the PIV measurements need to be carried out both vertically and horizontally in different positions. With cycle-to-cycle variations, observed in [7], numerous laser sheets need to be introduced at the same time. This creates a high complexity to capture double-exposure pictures on a respective laser sheet when all are introduced into the combustion chamber. Instead, the clouds of glowing soot particles that are created during combustion can be directly traced in the PIV software. By comparing two pictures at a time, the glowing particles are traced using cross-correlation. The light from the glowing particles is the tracer that the PIV software traces, and no form of extra seeding or laser-layer is used. In this way, the movement in the x-y- plane can be traced with information from not just one thin layer, but from a line of sight. Of course, this method makes it difficult to calculate the total velocity vector when the $\mathrm{z}$-axis is not included. To create an understanding of the in-cylinder movement and for later CFD comparison it is faster with the total information (instead of one thin layer). A better understanding of the entire flow field behaviour can be implemented. As will be shown later, the results for SN are quite stable, indicating that enough glowing soot particles remain in sight between the pictures. The total resolution of the pictures from the camera is $256 \times 256$ pixels, with a colour depth of 14 bit. The time delay between every picture, $\Delta \mathrm{t}$, was set to $28 \mu \mathrm{s}$, which means $0.168 \mathrm{CAD}$ at $1000 \mathrm{rpm}$. In Figure 5 and Figure 6 , the principle of the picture evaluation can be seen. Every picture was divided into 16x16 pixels integration windows in which a mean velocity vector

$$
|V|=\sqrt{V_{x}^{2}+V_{y}^{2}}
$$

was calculated between two pictures (at $t$ and $t+\Delta t$ ) for every integration window. For the next picture evaluation the next picture pair were evaluated. A film with velocity vectors was thereby created. To reduce the error reading from the pictures, DaVis built-in median filter and sliding average filter were used to fill up missing or incorrect vectors in the pictures. The missing/incorrect vectors mainly came from limited numbers of discernible particles in the area that could be tracked by DaVis. In Figure 6, the result of two evaluated pictures taken with 0.168 CAD between the exposures can be seen. The data for the vector arrows is just from those two pictures. The vector field, as seen, is what the observer can see of the movement, and the traceable particles are well captured by the PIV program. The program traces both the dark spots and the bright particles.

To reduce the influence of potential pressure fluctuations, which might give velocity vectors that are pointed in the direction of the pressure wave, an average vector field was created from 20 pictures. The time interval between ten frames represents one period of the pressure fluctuation (seen in the pressure trace in Figure 5), and with the average this error can be neglected when pictures with velocity vectors are plotted. The pressure oscillations are created by the premixed combustion, and at long ignition delay this can give large pressure oscillations that disturb the data evaluation. In an optical engine with single combustions, the ignition delay is longer

Page 6 of 17 
than for a continuously firing engine, since no residual gases remain in the cylinder and the surfaces in the combustion chamber have lower temperatures, which increases the ignition delay, see [2].

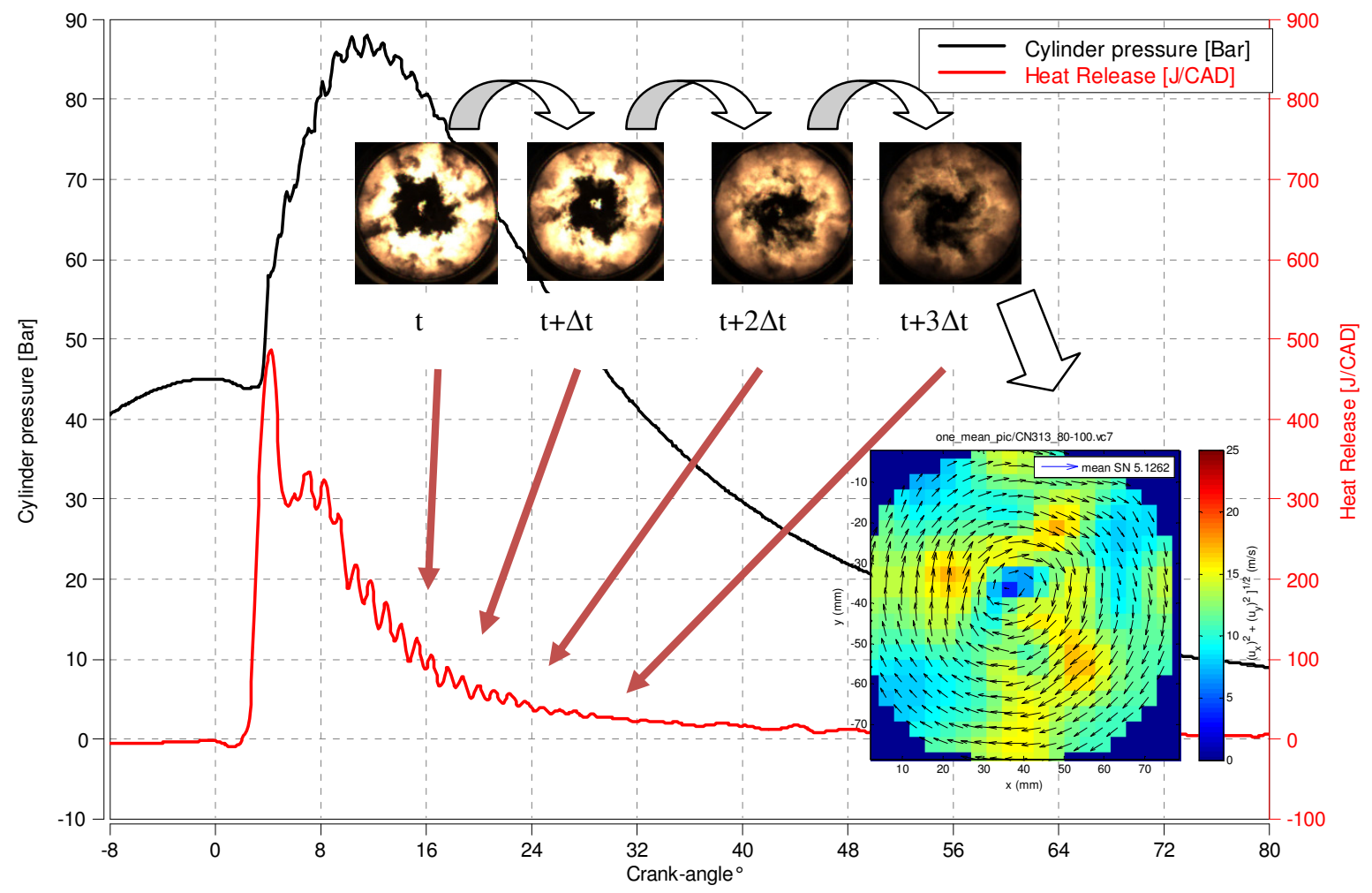

Figure 5 Load point $1 C$ at SN 3.4, cylinder pressure and heat release together with pictures of the burning particles that were evaluated with the PIV software.

From the PIV software, the velocity vectors were exported to MATLAB where further calculations were made. To calculate the SN from the velocity vectors some assumptions were needed. The tangential part from the geometrical centre, seen in Figure 6, of every velocity vector was estimated to give a contribution to the total rotational velocity in the cylinder. The tangential part of the velocity vector is calculated with:

$$
\bar{e}_{\perp}=\frac{\bar{e}_{z} \times \bar{r}_{i}}{\left|\bar{r}_{i}\right|}
$$

where

$\bar{e}_{z}=$ Unit vector along the cylinder axis $(0,0,1)$

$\bar{r}_{i}=$ radialvectorcoordinate $(\mathrm{x}, \mathrm{y}, \mathrm{z})$

$\bar{u}_{i}=\operatorname{velocity}$ vector $\left(\mathrm{u}_{\mathrm{x}}, \mathrm{u}_{\mathrm{y}}, \mathrm{u}_{\mathrm{z}}\right)$

, where $u_{z}$ is set to zero due to no information in the z-axis (2D camera shots). The angular velocity is then calculated:

$$
\omega_{i}=\frac{\bar{u}_{i} \cdot \bar{e}_{\perp}}{\left|\overline{r_{i}}\right|}
$$

where

$\omega_{i}=$ angular velocity $[\mathrm{rad} / \mathrm{s}]$

Angular velocities above $6000 \mathrm{rad} / \mathrm{s}$ and below $-1000 \mathrm{rad} / \mathrm{s}$ were assumed to be unrealistic and excluded from the SN calculations. 
From the angular velocities in the cylinder, the mean SN can be calculated by:

$$
S N_{\text {mean }}=\frac{\sum_{i=1}^{n} \omega_{i}}{\omega_{\text {eng_sp }} \cdot n}
$$

where

$n=$ number of angular speed vectors

$\omega_{\text {eng_sp }}=$ Enginecrankshaft angular vdocity $[\mathrm{rad} / \mathrm{s}]$
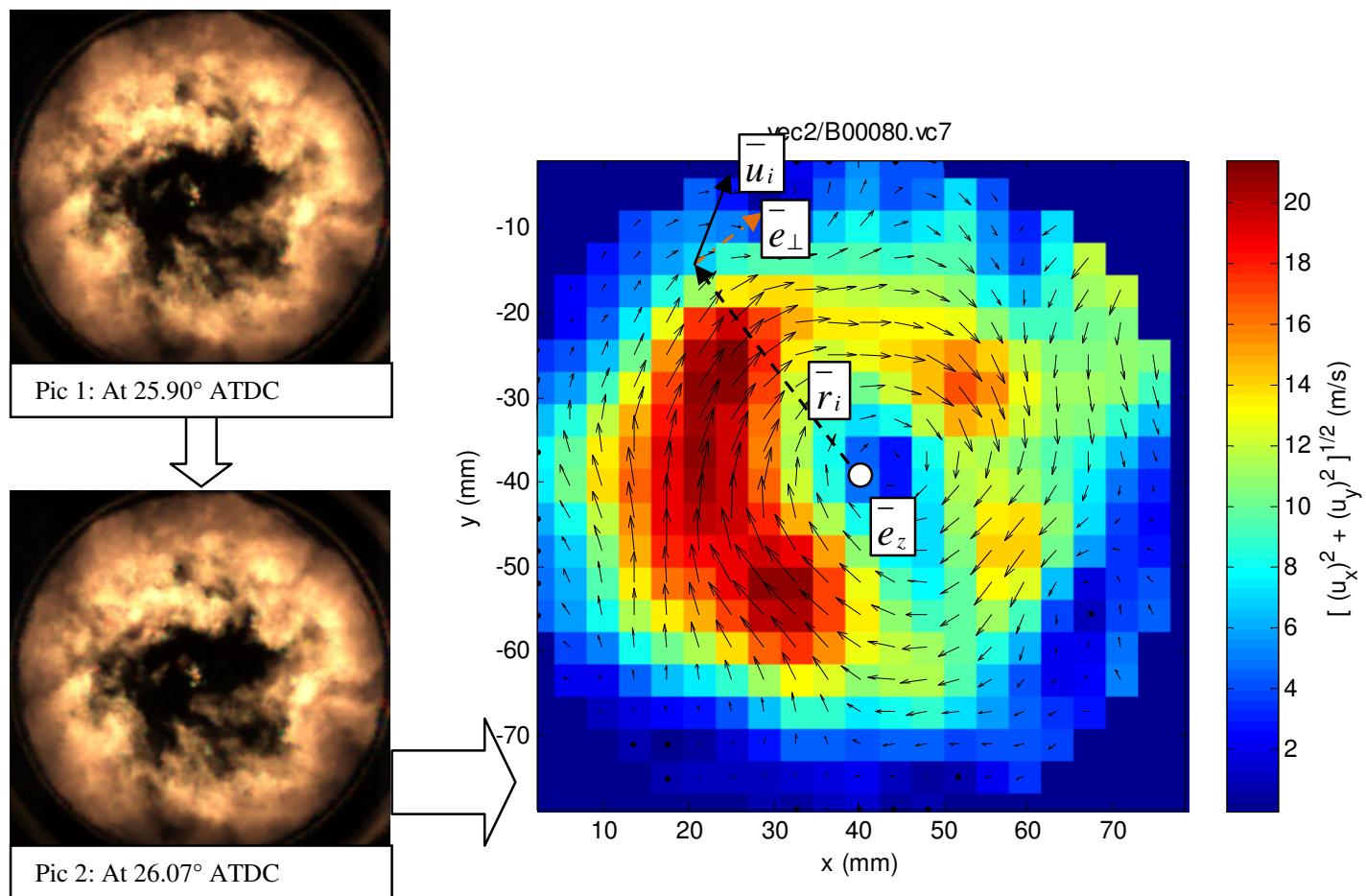

\section{Figure 6 Evaluated combustion pictures at 25.9 and $26.1^{\circ} \mathrm{ATDC}$ together with the calculated vector fields. In the vector field picture, the coordinate system is shown for SN calculation.}

To calculate the tumble flow the vectors are summed up:

$$
\begin{gathered}
\bar{u}_{x}=\frac{\sum_{i=1}^{n} u_{x, i}}{n}, \quad \bar{u}_{y}=\frac{\sum_{i=1}^{n} u_{y, i}}{n} \\
\text { Tumblespeed }=\sqrt{\left(\bar{u}_{x}\right)^{2}+\left(\bar{u}_{y}\right)^{2}}
\end{gathered}
$$

The tumble angle is then calculated:

$$
\text { Tumbleangle }=\arctan \left(\frac{\bar{u}_{y}}{\bar{u}_{x}}\right)
$$

\section{OPTICAL ENGINE TEST}

In the optical engine, the three load points seen in Table 1, were repeated from the six-cylinder engine tests. The light from the combustion was recorded with the camera and the aperture, together with the exposure time, and were chosen to limit the exposure of the pictures during combustion. The camera was synchronised to the engine with the injection signal. The engine was first motored without combustion. Boost pressure, back pressure, engine- and inlet-air temperature were set and stabilised at the same levels as in the six-cylinder engine. One injection was then performed which triggered the camera, and a movie was recorded with a time period of $28 \mu \mathrm{s}$ between the exposures. The DaVis PIV software was then used to evaluate the combustion pictures and the resulting vectors were calculated as described earlier. In Figure 7, load point 1c, with low SN head and flat piston, is plotted for one and two port Page 8 of 17 
operation. In this case, the calculated vectors are a mean value of 20 pictures during 3.36 CAD (roughly two pressure oscillation periods induced by the fast, premixed combustion). The injection ends at $9^{\circ}$ ATDC and the colour scale and arrow length in the pictures indicate the velocity. Deactivation of one port increases the mean SN from 2.67 to 4.11 in this case. The flow characteristic is more cylindrical in the high SN case, with well-defined centre point of rotation. The velocity is decreasing in the outer region compared with the inner piston bowl region. Flow from the side of the pictures is observed, which means that flow in z-direction exists. 10 CAD later in the expansion, Figure 8, the two-port case has a more structured flow characteristic compared with the earlier plot shown in Figure 7. The mean SN is slightly higher and it should be related to a better organisation of the swirling flow. The velocity gradients have decreased and are specially observed in high SN case. The small-scale vortexes have decreased and the bigscale vortex, swirl, dominate the picture even more.
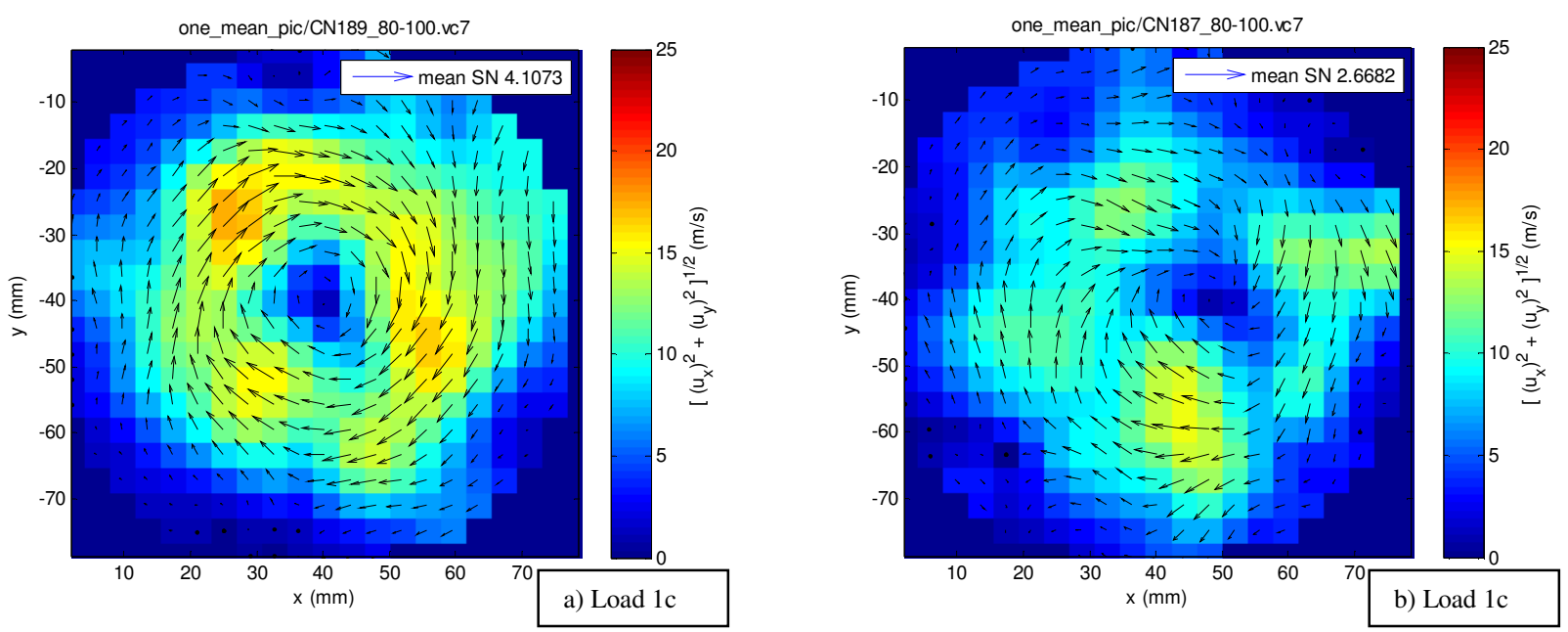

Figure 7 Time-averaged velocity vectors at $16.5^{\circ}$ to $19.9^{\circ} \mathrm{ATDC}$ for one port (a) and two ports (b) with flat piston and 1500 bar injection pressure.
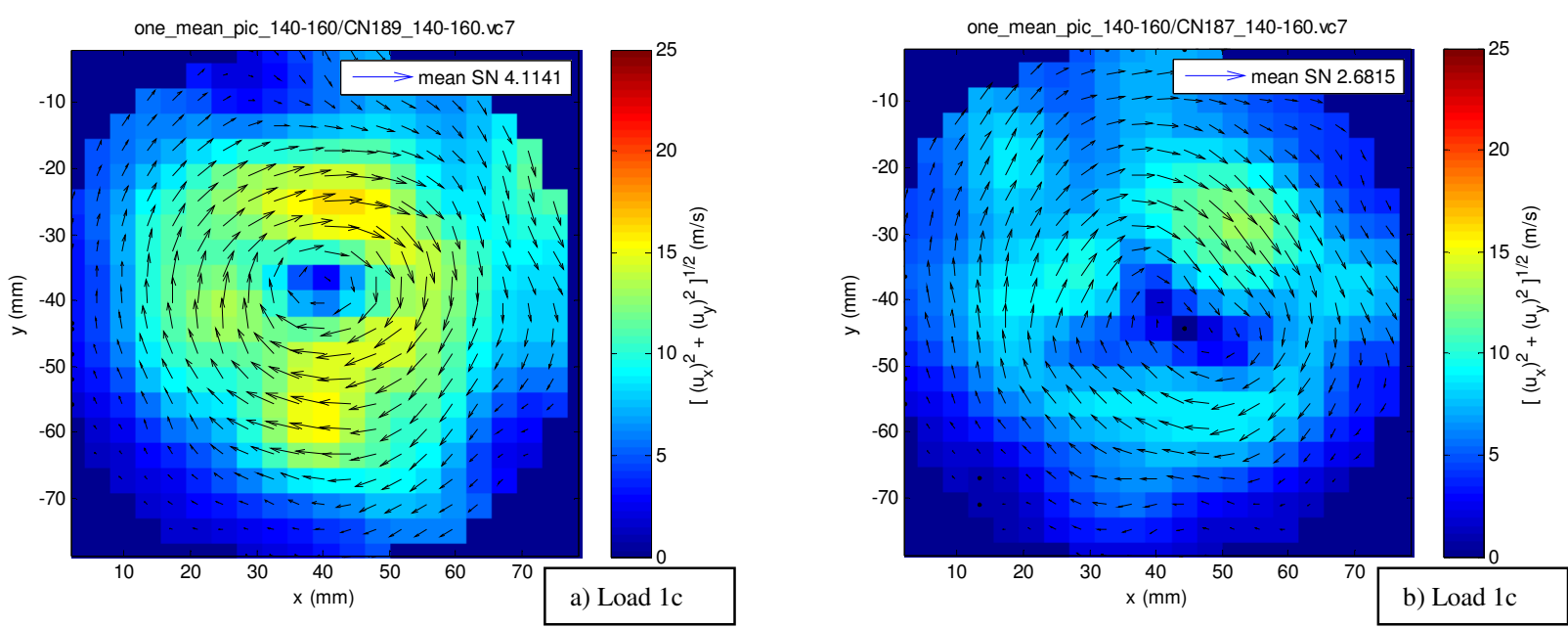

Figure 8 Time-averaged velocity vectors at $26.5^{\circ}$ to $29.9^{\circ}$ ATDC for one port (a) and two ports (b) with flat piston and 1500 bar injection pressure.

In Figure 9, the angular velocity profiles are plotted for the cases shown in Figure 7 and Figure 8. As in the previous figures, timeaverage (average over 3.4 CAD) velocity vectors are used. In the legend, the mean SN is calculated with equation 9 for each respective case. The angular velocity profiles are calculated from the tangential velocity of every velocity vector and a mean value is calculated for respective radial position. It is clear to see that the angular velocity is higher in the centre region compared with the outer piston bowl region. When one port, is used the deviation of angular velocity in the centre region is even greater compared with the outer region. The deviation in angular velocity is larger at earlier CAD (solid lines in Figure 9), compared with the later CAD (dotted lines in Figure 9). The difference in angular velocity seems to decrease with later CAD in both tested cases, with a slightly increased velocity at the outer bowl region (between $15 \mathrm{~mm}$ to $30 \mathrm{~mm}$ in the picture) and a lower velocity at the inner region (between $5 \mathrm{~mm}$ to $15 \mathrm{~mm}$ ).

Page 9 of 17 


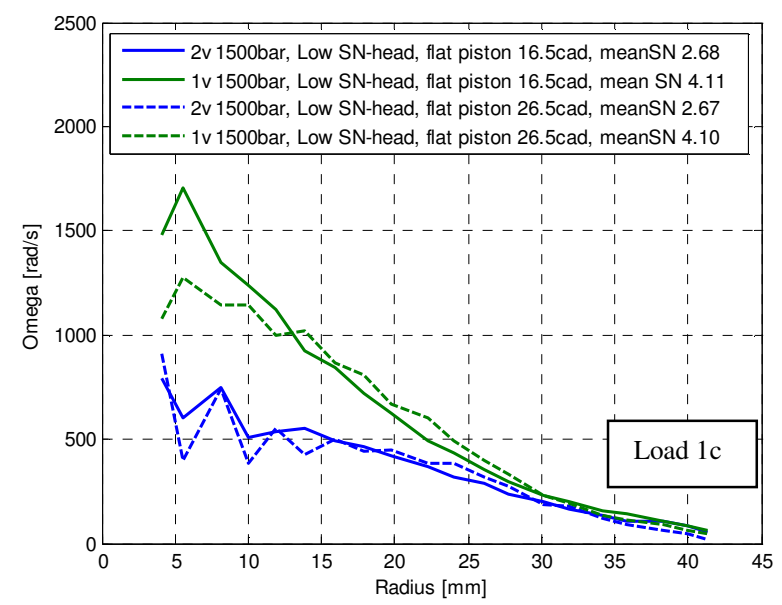

Figure 9 Angular velocity profile versus radial position in a flat piston bowl.

In Figure 10, the mean $\mathrm{SN}$ is plotted for load point 1c with the flat piston and low SN-head. The injection ends at $9^{\circ}$ ATDC. Before that, the data is not reliable due to the injection event that creates lots of turbulence. Two pictures are evaluated for every data point and used to calculate the velocity vectors. Equation 9 is used to calculate the SN at every sampled CAD. No mean value from several pictures are used in this case and some fluctuations in the graph are observed. It is clear to see that after the injection event has ended, the calculated SN starts to increase. The traced glowing soot particles can be assumed to be small, light and follow the flow field well. The injection creates lots of flow in the z-direction, which we cannot see with this evaluation method. When this injection-induced turbulence dies out (as seen on the unprocessed combustion pictures), we see the acceleration of the flow field in the swirl direction that can be related to differences in the z-direction that are smoothed out. Big-scale turbulence is transformed into small-scale turbulence very quickly. If we examine one small-scale turbulence vortex, on one turnover the turbulence has lost most of its energy and the length scale is significantly smaller [18]. The total angular momentum on the big-scale vortex (swirl) can be assumed to be the same before and after the injection. With the added fuel mass under the injection event, the angular velocity is thereby decreasing with conserved total angular momentum. The one-port case has a constantly higher SN compared with the two-port case. The SN is then quite constant from $15^{\circ}$ ATDC until $30^{\circ}-35^{\circ}$ ATDC, when the observed SN decreases. The SN decreases under the influence of the geometrical change in the cylinder. Gas flows into the squish region and more mass is placed on the large radius. With the assumption that the total angular momentum in the cylinder is conserved, it gives a natural decrease in angular velocity. At late CAD, when even more gas is placed in the squish plane, which cannot be observed in this test set-up $(80 \mathrm{~mm}$ observation glass and $130 \mathrm{~mm}$ cylinder bore), this creates a problem in calculating the total momentum of the gas in the cylinder. This method, with this test set-up, is therefore most reliable at CAD close to TDC. Another factor that reduces the SN is the friction to the cylinder walls.

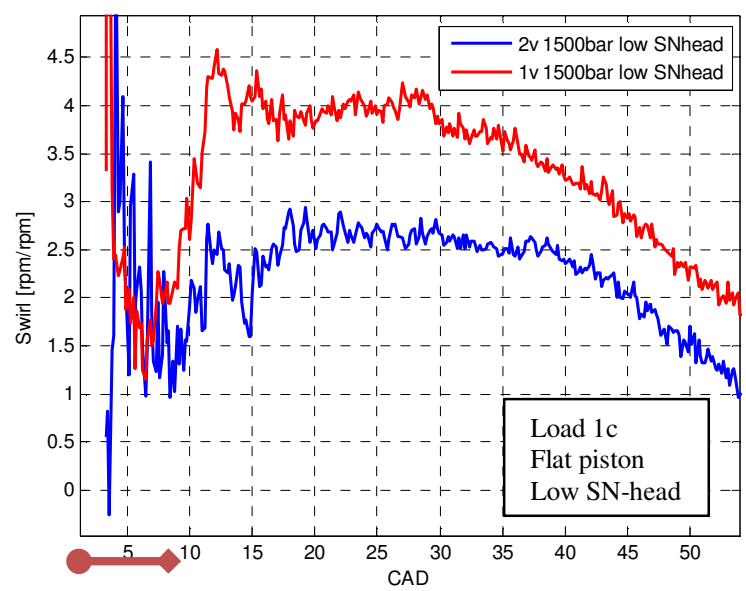

Figure 10 SN versus CAD in the after-oxidation period. The red line with the round circle and rhomb indicates the injection period.

The tumble velocity and tumble angle observed from the pictures are plotted in Figure 11 for load point $1 \mathrm{c}$. It is clear to see that the tumble velocity decreases rapidly. The case with high calculated tumble in GT-POWER, 1v, has a higher tumble velocity compared with the low tumble case, $2 \mathrm{v}$, as estimated in GT-POWER. The rotation of the tumble angle follows the SN in the $1 \mathrm{v}$ case. The

Page 10 of 17 
rotation is a little slower than expected, but is in the same direction as the swirl. The $2 \mathrm{v}$ case angle does not follow the swirl, but the tumble velocity is so slow that tumble can be expected to be erased. The global tumble vortex is thereby transformed to smaller scales, not only under the compression but also slightly after TDC.
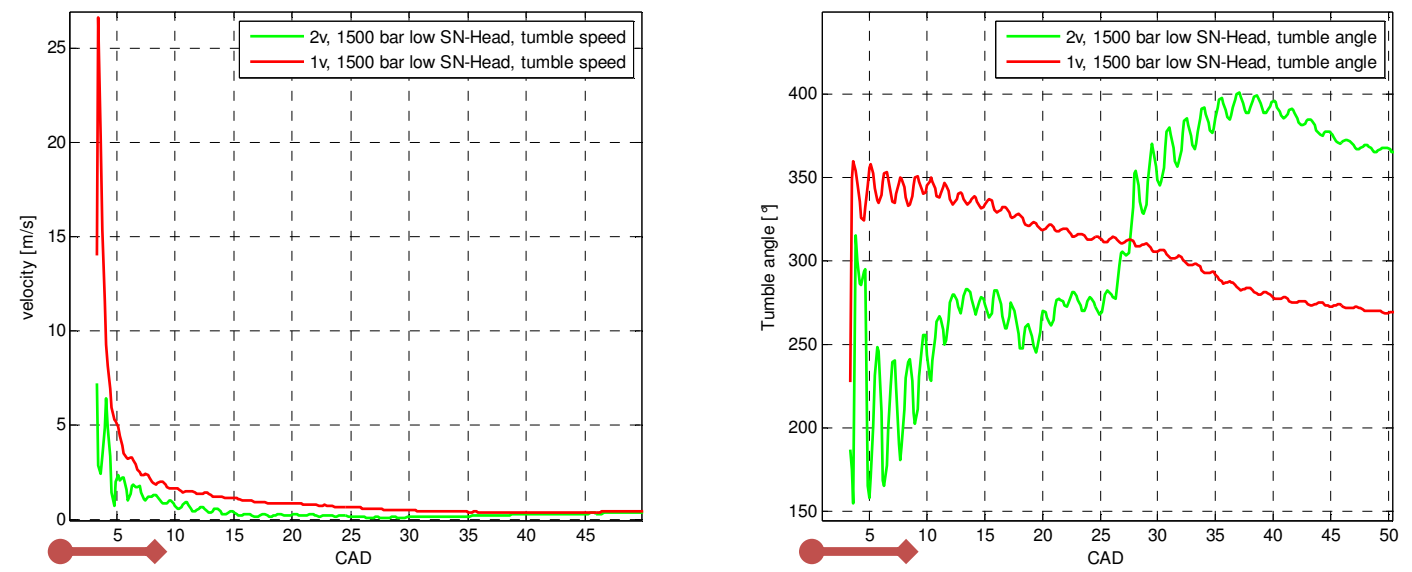

Figure 11 Tumble velocity and tumble angle plotted versus CAD. The injection event ends at 9 CAD and is marked in the figure.

When the piston bowl-shaped glass was used together with the high SN head, the injection pressure was varied from 500 to 1500 bar, with increments of 500 bar. One obvious trend that was observed is that the SN increases with injection pressure. In Figure 12, Figure 13 and Figure 14, load point 1a, b, c (injection pressure 500, 1000 and 1500 bar) velocity vectors are shown for one- and two-port operation. The calculated SN, defined in equation 9, is higher in the one-port cases (pictures to the left), but is also higher with higher injection pressure. The swirl centre is generally more off-centre compared with the two port cases, as seen in the following pictures. The tumble number is higher in the one-port case, 2.2 at BDC compared with 1.1 at BDC for two ports, see Table 4 . The tumble flow disturbs the swirling flow and tilts the swirl centre axis. Some of the tumble flow is transformed into small-scale turbulence during compression, but the off-centre location of the swirl centre, also observed in [5], survives the compression and the combustion, as seen in the following figures. In the case with the flat piston bowl and low SN-head, the trend was opposite. With two ports, the swirling flow had less structure that did not rotate around a swirl centreline. Unstructured flow makes it harder to organize the flow around a centre-point, as observed in [5]. Low SN gives less structure. This can be one explanation of why the trend is opposite to the high SN cases with piston bowl shaped glass. With the shaped piston bowl, the velocity nearest the circumference is higher and the velocity distribution is more homogenous compared with the flat piston cases. Some of these trends can be related to the lower flow velocity with a low SN head, but the bowl-shaped piston helps to organise the rotational flow with less mass in the middle of the combustion chamber. Another observed trend is that higher velocity gradients are observed at higher injection pressures. The increased sprayinduced small-scale turbulence increases the velocity gradients after the end of the injection, shown in the pictures with red fields (high velocity vectors). Flow in the z-plane exists and gives local velocity differences.
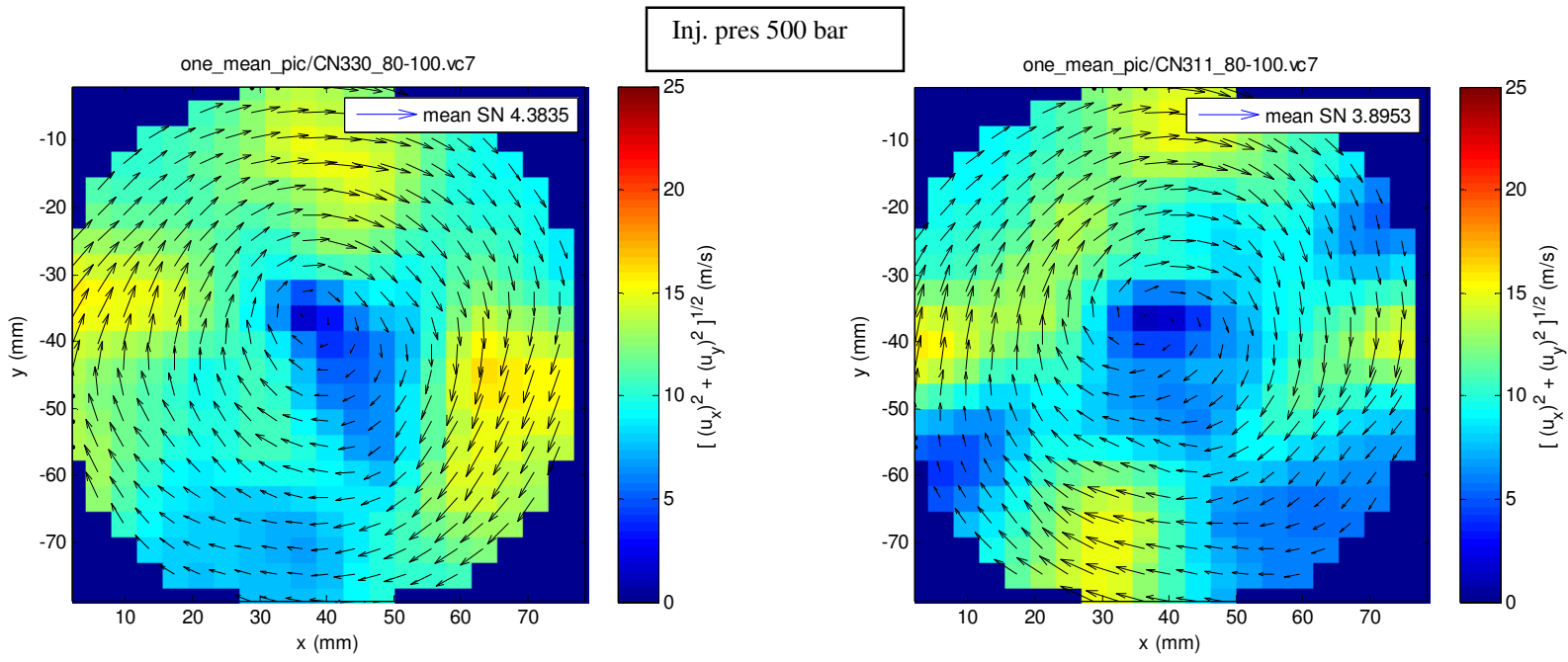

Figure 12 Velocities for the bowl-shaped piston at $16.5^{\circ}$ to $19.9^{\circ}$ ATDC and injection pressure 500 bar. The picture on the left shows the single-port case and the picture on the right shows the two-port case. 

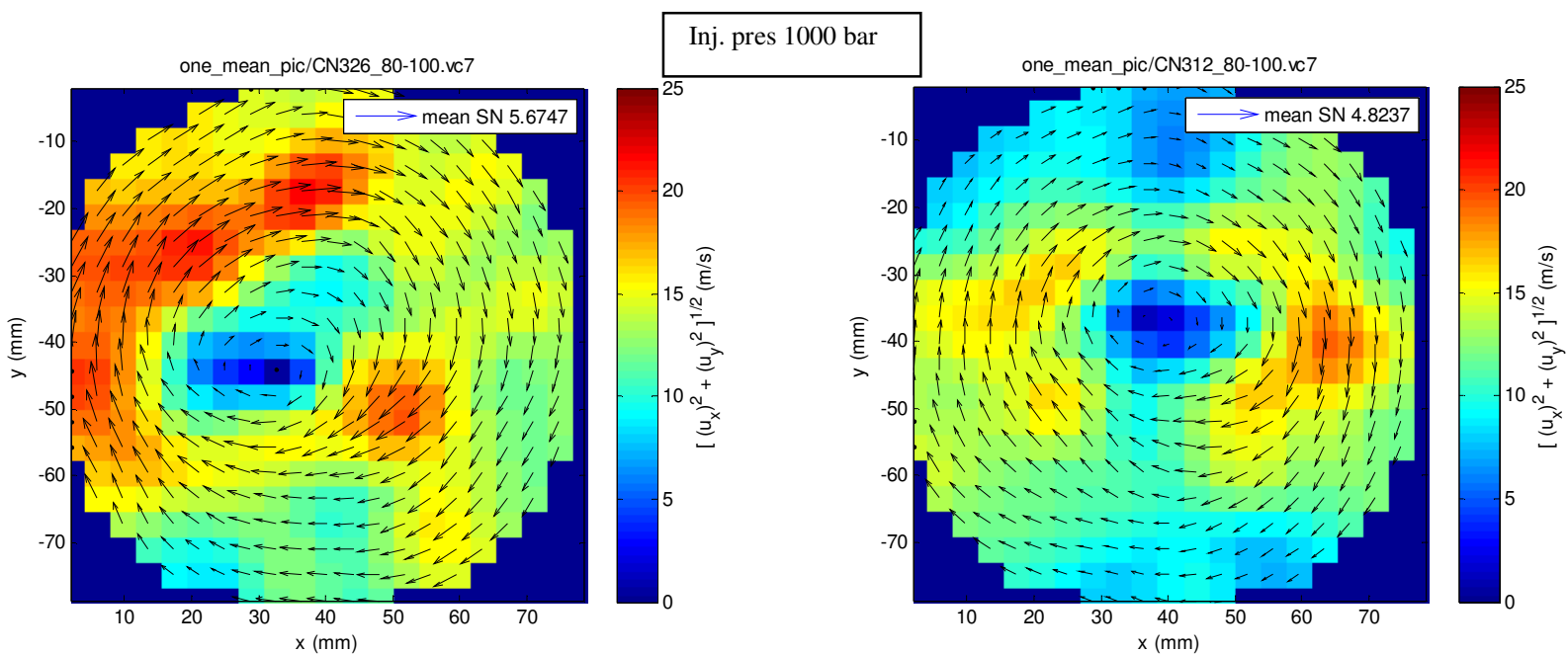

Figure 13 Velocities for the bowl-shaped piston at $16.5^{\circ}$ to $19.9^{\circ}$ ATDC and injection pressure 1000 bar. The picture on the left shows the single-port case and the picture on the right shows the two-port case.
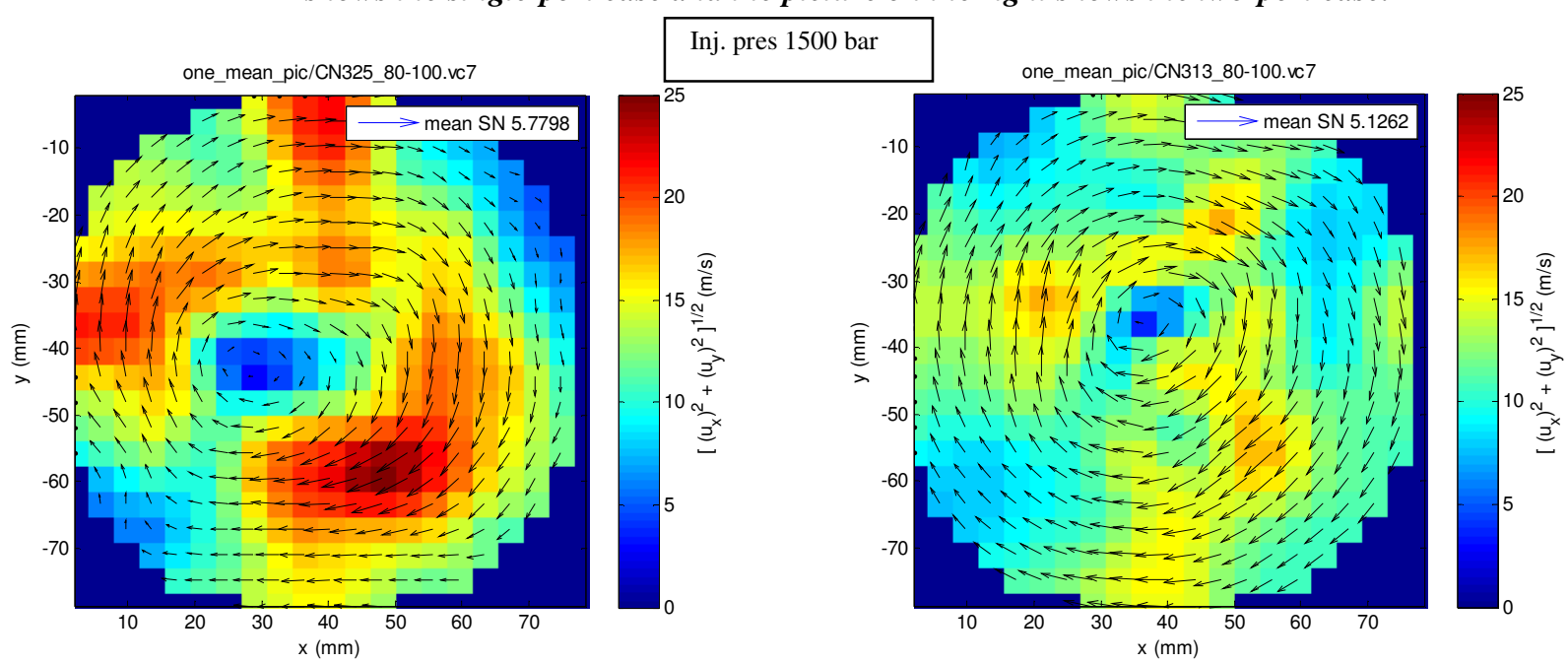

Figure 14 Velocities for the bowl-shaped piston at 16.5 to $19.9^{\circ}$ ATDC and injection pressure 1500 bar. The picture on the left shows the single port-case and the picture on the right shows the two-ports case.

In Figure 15 and Figure 16, load points 2 and 3 are plotted for one- and two-port operation. The higher load, 13 bar IMEP in loadpoint 2 and 20 bar IMEP in load-point 3, shows the same trend as in load point 1a, b, c, with more off centre flow under one port operation. With one-port it is also seen that velocity vectors in the centre region are longer and differ more compared with the vectors in the outer piston bowl region. Higher swirl increases the velocity difference over the bowl radii. The earlier observations regarding increased velocity gradients with increased injection pressure still exist when the load is also amplified together with injection pressure. 

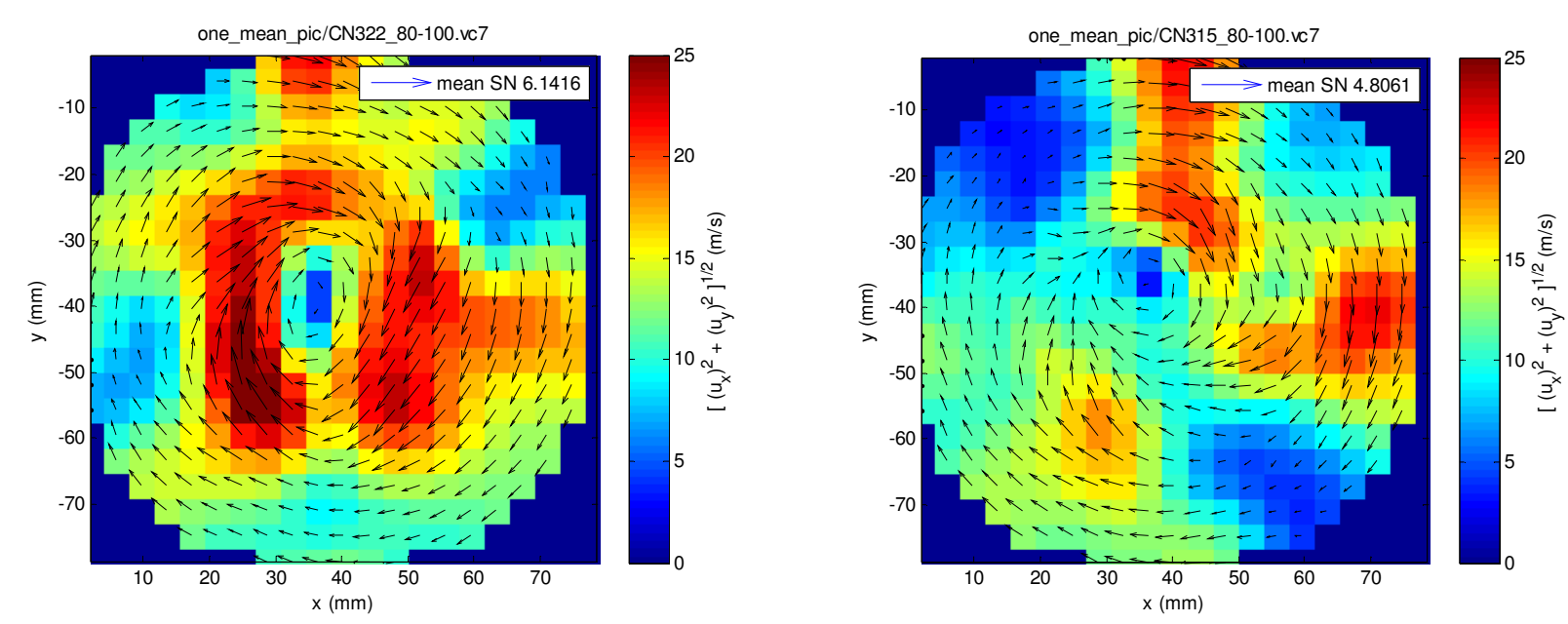

Figure 15 Velocities for the bowl-shaped piston at 16.5 to $19.9^{\circ}$ ATDC and injection pressure 2000 bar. The picture on the left shows the single-port case and the picture on the right shows the two-port case. Load point 2.
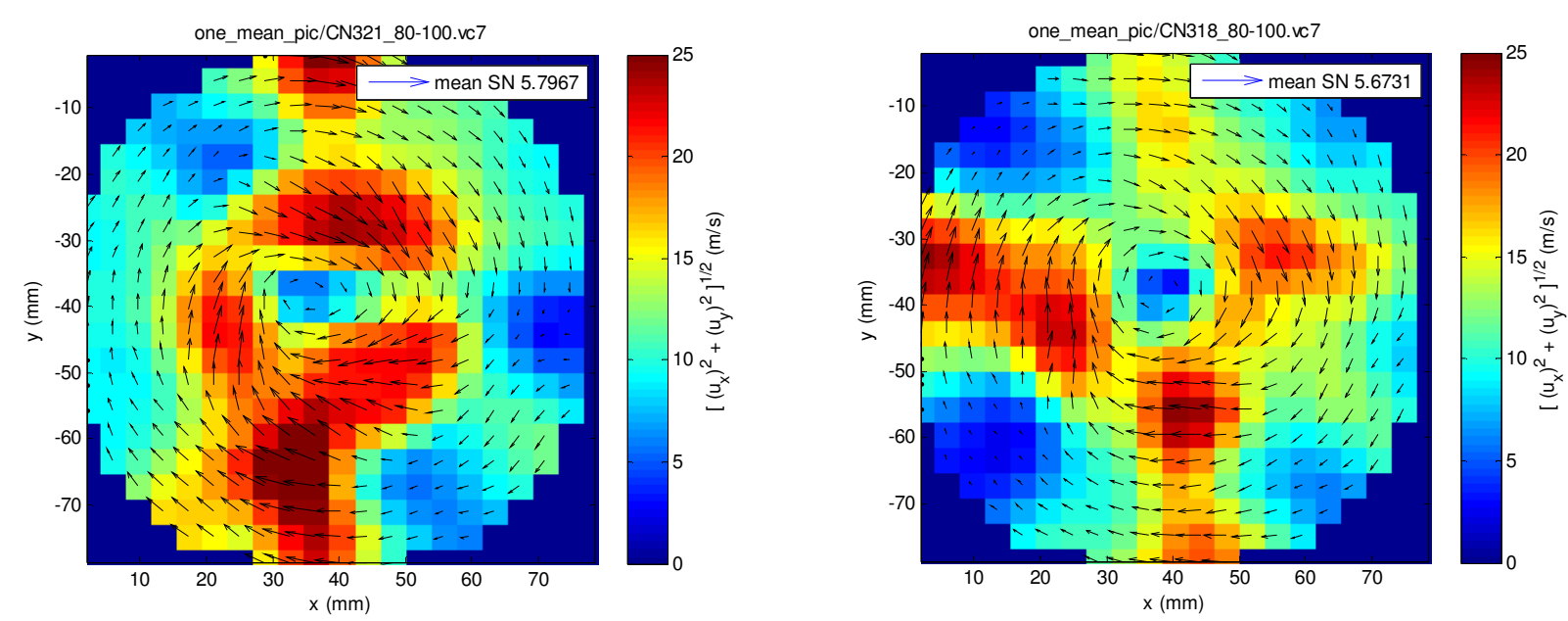

Figure 16 Velocities for the bowl-shaped piston at 16.5 to $19.9^{\circ}$ ATDC at injection pressure 2500 bar. The picture on the left shows the single-port case and the picture on the right shows the two-port case. Load point 3.

In Figure 17, the angular velocity distribution is plotted for above showed load cases with the bowl-shaped piston and high SN-head. Observe that load 1a, b, c together with loads 2 and 3 are plotted on the same graph. Clearly, the difference in angular velocity at different radii can been seen. The angular velocity is higher in the middle of the combustion chamber compared with the piston bowl circumference. The trend is clearly increasing with higher injection pressures. When the SN is increased by deactivating one port, the differential trend in angular velocity at different radii remains at higher injection pressures. The differential in angular velocity along the bowl radius improves mixing when the turbulence gives increased vorticity and the unburned and burned gases mix faster under after oxidation part of combustion. The reason why the angular velocity deviates more at higher injection pressure can be found in fuel that is injected at higher pressure which is forced to the outer piston bowl region with increased force. In other words, the penetration depth is longer at higher injection pressure. The air angular momentum can be assumed to be relatively constant up to TDC before fuel injection starts between the tested cases. When the fuel reaching the outer bowl region, the air that is there needs to accelerate the fuel mass. The observed diameter is $80 \mathrm{~mm}$, of the total cylinder bore of $130 \mathrm{~mm}$, this makes the observed volume at TDC $\sim 90 \mathrm{~cm}^{3}$ of a total volume of $\sim 125 \mathrm{~cm}^{3}$. The calculated mean SN is therefore higher for higher injection pressures when the non-observable outer region is not taken into account. In the low injection pressure case, fuel is more uniformly distributed over the piston bowl when the penetration length is restricted, which is also observed in [9]. The injection time is also longer for low injection pressures for the same amount of fuel, this also makes the distribution in the piston bowl more uniform. The fuel-spray itself influences the aerodynamics in the cylinder when it is injected perpendicular to the flow. Most of the swirling airflow (which, at present, does not contribute to the combustion) needs to pass the fuel sprays and the combustion cloud that is creating drag. A longer injection period means a longer time for the fuel spray core to influence the air swirling flow dynamics. In the case with 2500 bar injection pressure and load 20 bar 
IMEP, the observed mean SN decreases. The reason for this is that the lambda are decreased in this test. The conserved airflow momentum in the cylinder needs to accelerate more fuel mass, which gives a decreased SN.
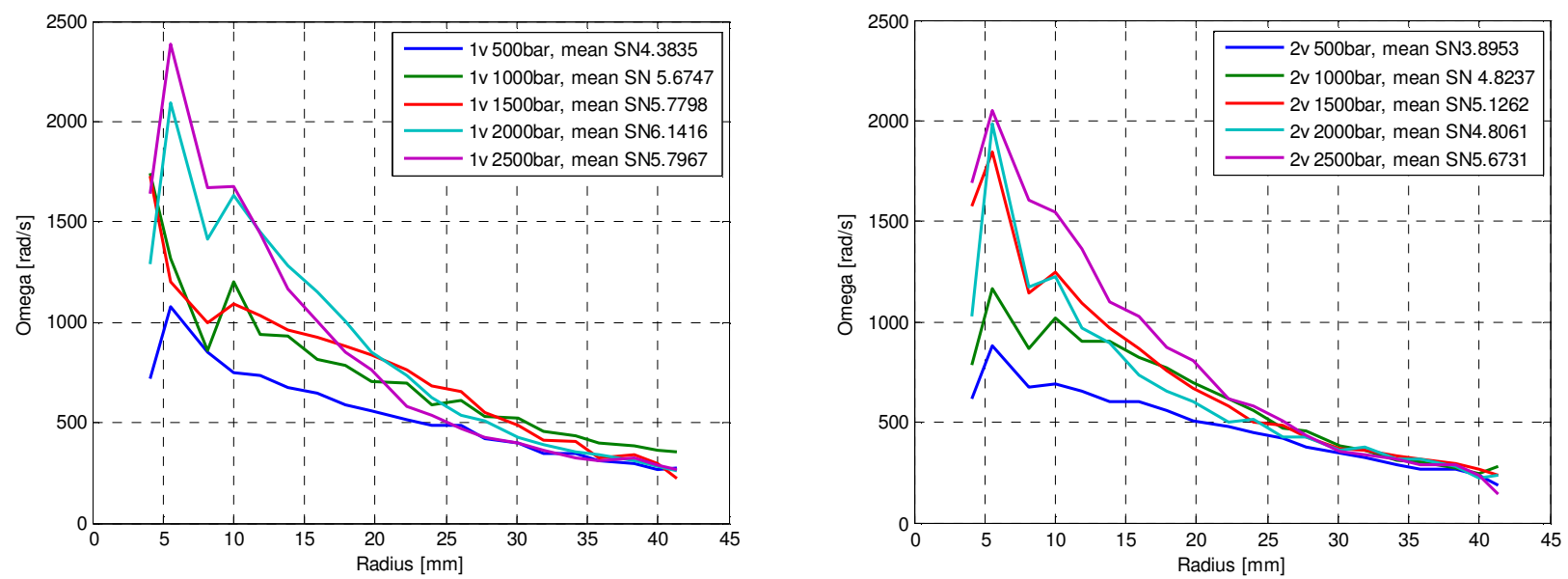

Figure 17 Angular velocity at 16.5 to 19.9 CAD for one-port in the left-hand picture and two-ports in the right-hand picture. In the legend, mean SN is calculated with equation 9 for each respective case. At 500 bar to 1500 bar, the load is 10 bar IMEP, at 2000 bar injection pressure, load is 13 bar IMEP and at 2500 bar inj. pres. the load is 20 bar IMEP.

The differential of angular velocity at different radii is partly reduced at 10 CAD later, as seen in Figure 18 the same trend as observed in Figure 9. The shear stress through the velocity profile together with the turbulence eddies had time to equalise at the different radii due to dissipation. The mean SN at one-port operation decreases faster compared with the two-port case. The reason is, of course, because that the SN is higher in the one-port case and drops from a higher level, which gives higher flow losses.
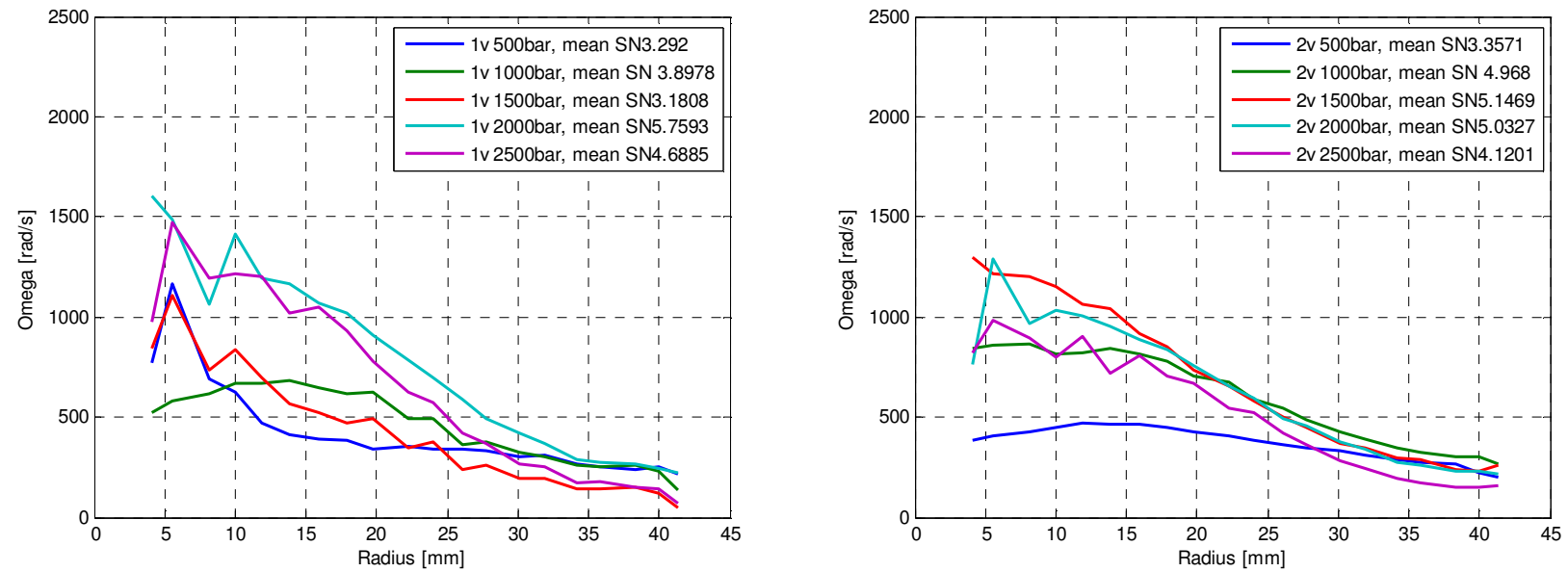

Figure 18 Distribution of angular velocities for the bowl-shaped piston, one (left picture) and two (right picture) ports between 26.5 and $29.9^{\circ}$ ATDC. At 500 bar to 1500 bar the load is 10 bar IMEP, at 2000 bar inj. Load is 13 bar IMEP and at 2500 bar inj. Pres. the load is 20 bar IMEP.

Finally, the results from GT-POWER and the empirical expression from [17] (called "CFD-based") are compared with the cross correlation, from combustion pictures, in Table 5 and Figure 19. At low SN, the calculations from GT-POWER and CFD-based calculations at TDC are quite equal. However, at a higher SN the two methods start to differ significantly, see Table 5. The comparison with GT-POWER is done after fuel injection, and it can be seen that, even in this case, GT-POWER differs more at a higher SN. The CFD-based method cannot fully be compared with the measured values, since it does not take the fuel injection into account. Our measurements are done after fuel injection with increased mass in the cylinder for a given angular momentum induced under the inlet stroke. But the CFD-based method seems like it underestimates at high a SN, as it indicates a lower SN at TDC compared with measured SN at 16.5 CAD (high SN-head cases). GT-POWER significantly overestimates the highest SN (high SNhead 1v). However, with two ports and a high SN head, it seems that GT-POWER catches the trend better even if it indicates a higher

Page 14 of 17 
SN than measured here, see Table 5. If we assume that the total angular momentum is the same before and after the injection, with the added fuel mass under the injection event, the angular velocity should therefore decrease with conserved total angular momentum. Compared with the calculated SN in GT-POWER, Table 5, 3.54 (with combustion in GT-P) the measured SN is lower, 2.69 in the two-port case.

\begin{tabular}{|c|c|c|c|c|c|c|c|c|}
\hline \multirow[b]{2}{*}{ Swirl number } & \multicolumn{4}{|c|}{ GT-POWER } & \multirow{2}{*}{\begin{tabular}{|c} 
CFD based \\
TDC
\end{tabular}} & \multicolumn{3}{|c|}{ Cross corr. combustion pictures } \\
\hline & BDC & TDC & $16.5^{\circ} \mathrm{ATDC}$ & $26.5^{\circ} \mathrm{ATDC}$ & & \begin{tabular}{|c|}
$16.5^{\circ}$ to \\
$19.9^{\circ}$ ATDC
\end{tabular} & $\begin{array}{c}26.5^{\circ} \text { to } \\
29.9^{\circ} \text { ATDC }\end{array}$ & $\begin{array}{c}16.5^{\circ} \text { to } \\
26.5^{\circ} \text { ATDC }\end{array}$ \\
\hline $\begin{array}{c}\mathrm{v} \text { flat piston, low SN- } \\
\text { head } \\
2 \mathrm{v} \text { flat piston low SN- }\end{array}$ & 2.3 & 4.00 & 3.87 & 3.78 & 3.21 & & & \\
\hline head (with combustion) & 2.3 & 4.00 & 3.7 & 3.54 & 3.21 & 2.67 & 2.68 & 2.69 \\
\hline $\begin{array}{c}\text { 1v flat piston, low SN- } \\
\text { head } \\
1 \mathrm{v} \text { flat piston, low SN- }\end{array}$ & 4.19 & 7.10 & 6.74 & 6.55 & 4.43 & & & \\
\hline head (with combustion) & 4.19 & 7.00 & 6.43 & 6.15 & 4.43 & 4.11 & 4.11 & 4.2 \\
\hline $\begin{array}{l}\text { 2v bowl shaped piston, } \\
\text { high SN-head } \\
\text { 2v bowl shaped piston, } \\
\text { high SN-head (with } \\
\text { combustion) }\end{array}$ & 3.4 & 6.17 & 6.09 & 6.04 & 4.00 & 5.13 & 5.15 & 5.14 \\
\hline $\begin{array}{l}\text { 1v bowl shaped piston, } \\
\text { high SN-head } \\
\text { 1v bowl shaped piston, } \\
\text { high SN-head (with } \\
\text { combustion) }\end{array}$ & 6.3 & 9.80 & 9.69 & 9.53 & 5.25 & 5.78 & $\begin{array}{c}\text { No } \\
\text { measureme } \\
\text { nt }\end{array}$ & 5.34 \\
\hline
\end{tabular}

In Figure 19, the SN at BDC (from GT-POWER) is plotted against SN TDC for the different methods. It is seen that the measured SN from PIV pictures done in this work and the CFD-based SN, equation 4, from [17], follow the same trend. At a higher SN at BDC, the SN at TDC starts to flatten out. The CFD-based SN is calculated at TDC without injected fuel compared with the measured values that are at $16.5^{\circ}$ ATDC with much injected fuel mass that reduces the flow velocity. The comparison shows that the CFD-based method underestimates the measured values. GT-POWER seems to have a more linear model and it deviates a lot at high SN compared with the other two methods.

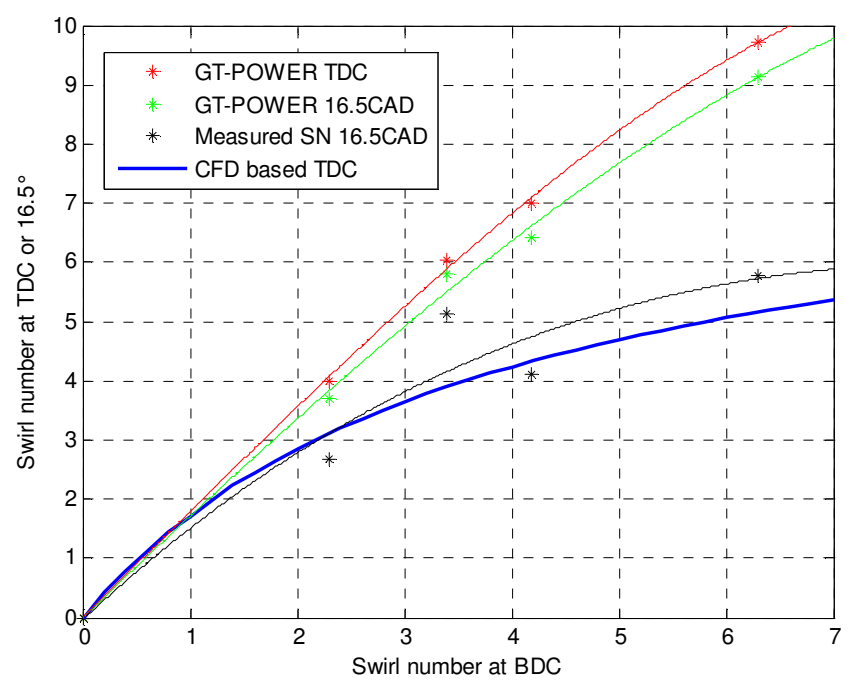

Figure 19 Comparison between the two different SN models, GT-POWER and CFD-based, with measured SN in the optical engine. 


\section{SUMMARY/CONCLUSIONS}

This paper has shown that the large-scale in-cylinder flow can be captured from the late combustion phase from illuminated soot particles. The flow structure at late combustion (after the injection has ended) in the cylinder has been evaluated with PIV software to calculate the velocity field by cross correlation. SN have then been calculated from the velocity vector fields and plotted against CAD for the after-oxidation part of the combustion. It has also been reported a comparison for SN between GT-POWER simulations and CFD-based correlation for TDC calculations with optical engine measurements.

1. The method has shown similar results as the CFD-based correlation method and good robustness on the evaluated data.

2. The angular velocity deviates at different radii in the after-oxidation period. No solid body rotation can be observed in the cylinder directly after injection has ended. Higher injection pressure gives increased angular velocity deviation between the gas in the middle of the combustion chamber and at the piston bowl boundary. The mixing in the after-oxidation period is improved due to the increased vorticity and turbulence that forces the gas to mix.

3. Off centre swirl flow observed before TDC in several of the above mentioned papers, survives the compression and combustion (shown in this paper). The diesel sprays that are injected symmetrically into the combustion chamber are thereby exposed unsymmetrical to the swirl.

4. Increased injection pressure gives higher velocity gradients in the after oxidation period. The increased spray-induced turbulence gives less uniform big-scale vortex and creates a lot of flow in z-direction.

\section{REFERENCES}

1. Aronsson, Ulf, et al. "Analysis of the Correlation Between Engine-Out Particulates and Local $\Phi$ in the Lift-Off Region of a Heavy Duty Diesel Engine Using Raman Spectroscopy”, SAE 2009-01-1357.

2. Dembinski, Henrik and Ångström, Hans-Erik. ”An Experimental Study of the Influence of Variable In-Cylinder Flow, Caused by Active Valve Train, on Combustion and Emissions in a Diesel Engine at Low $\lambda$ Operation” JSAE 201109083.

3. Suzuki, Takashi. "The Romance of Engines.”, 1997, ISBN 1-56091-911-6.

4. Antila, Eero, et al. "Effect on Intake Channel Design to Cylinder Charge and Initial Swirl", SAE 2010-01-0624.

5. Nordgren, Henrik, et al. "Comparison Between In-Cylinder PIV Measurements, CFD Simulations and Steady-Flow Impulse Torque Swirl Meter Measurements", SAE 2003-01-3147.

6. Deslandes, Willy, et al. "PIV Measurements of Internal Aerodynamic of Diesel Combustion Chamber", SAE 2003-01-3083.

7. Deslandes, Willy, et al. "Airflow Cyclic Variations Analysis in Diesel Combustion Chamber by PIV Measurements", SAE 200401-1410.

8. Petersen, Benjamin and Miles, Paul. "PIV Measurements in the Swirl-Plane of a Motored Light-Duty Diesel Engine", SAE 2011-01-1285.

9. Auriemma, M., et al. "Influence of a Swirling Airflow on an Evaporating Diesel Spray from a Common Rail Injection System under Realistic Engine Conditions“, SAE 2007-24-0021.

10. Lindström, Mikael and Ångström, Hans-Erik. "A Study of In-Cylinder Fuel Spray Formation and its Influence on Exhaust Emissions Using an Optical Diesel Engine",. SAE. 2010-01-1498.

11. Pires da Cruz, A., et al. "Turbulent Self-Ignition and Combustion Modelling in Diesel Engines", SAE 1999-01-1176.

12. Dembinski, Henrik and Lewis, Clive. "Miller-cycle on heavy duty diesel engines“, Stockholm: KTH, 2009. Master Thesis, MMK2 2009:1 MFM124.

13. Morel, Thomas and Keribar, Rifat. "A Model for Predicting Spatially and Time Resolved Convective Heat Transfer in Bowlin-Piston Combustion Chambers", SAE 850204.

14. Morel, T., et al. "Model for Heat Transfer and Combustion in Spark Ignited Engines and Its Comparison with Experiments", SAE 880198.

15. Uzkan, Teoman, et al. "Characterization of Flow Produced by High-Swirl Inlet Port", SAE 830266.

16. Yu R., et al. "Numerical and Experimental Investigation of Turbulent Flows in a Diesel Engine", SAE 2006-01-3436.

17. Pastor, J. V, et al. "A Methodology to Estimate the Swirl Number at TDC In DI Diesel Engines: Through the Combination of CFD and Steady Flow Rig Results“, SAE 2004-01-1876.

18. Pope, Stephen B. “Turbulent flows“, Cambridge University press year 2000, ISBN 978-0-521-59125-6 


\section{CONTACT INFORMATION}

Henrik Dembinski M.Sc.

Scania CV AB

Telephone: $+46(0) 855380769$

Fax: +46 (0)8 55381005

E-mail: henrik.dembinski@ scania.com

Postal address: SE-151 87 Södertälje, Sweden

\section{ACKNOWLEDGMENTS}

This work was supported by Scania CV AB, the Swedish Energy Agency, and the Royal Institute of Technology (KTH Stockholm). 